\title{
Conformal Field Theory and Hyperbolic Geometry
}

\author{
P. Kleban ${ }^{1,2,3}$ and I. Vassileva $\mathbf{~}^{2,4}$ \\ ${ }^{1}$ School of Mathematics \\ Institute for Advanced Study \\ Princeton, New Jersey 08540 \\ ${ }^{2}$ LASST and ${ }^{3}$ Department of \\ Physics \& Astronomy \\ University of Maine \\ Orono, Maine 04469* \\ ${ }^{4}$ Department of Mathematics \\ Univeristy of Massachusetts \\ Amherst, Massachusetts 01003
}

\begin{abstract}
We examine the correspondence between the conformal field theory of boundary operators and two-dimensional hyperbolic geometry. by consideration of domain boundaries in two-dimensional critical systems, and the invariance of the hyperbolic length, we motivate a reformulation of the basic equation of conformal covariance. The scale factors gain a new, physical interpretation. We exhibit a fully factored form for the three-point function. A doubly-infinite discrete series of central charges with limit $c=-2$ is discovered. A correspondence between the anomalous dimension and the angle of certain hyperbolic figures emerges.
\end{abstract}

* Present and permanent address. e-mail: kleban@maine.maine.edu 
In this letter, we establish several connections between the conformal field theory of boundary operators and two-dimensional hyperbolic geometry. First, by consideration of domain boundaries in two-dimensional critical systems, and the invriance of the hyperbolic length, we motivate a reformulation of the basic equation of conformal covariance. The scale factors gain a new, physical interpretation. They operate to keep the distance from the end of the domain boundary to the boundary of the system fixed. We also point out that for any geometry conformally equivalent to the half-plane, domain boundaries in twodimensional critical systems follow hyperbolic geodesics. Their energy per unit hyperbolic length is finite. Motivated by these results, we next exhibit a completely factored form for the three-point function (and the prefactor of the four-point function). Here, a connection between the anomalous dimension of a primary operator and the angle of a hyperbolic figure appears. Finally, we impose the condition that the Schwarz function defined by a four-point function of opertors degenerate at level two correspond to a hyperbolic tiling, or tessellation. this leads to a new, doubly-infinite discrete set of minimal models. The angle-dimension correspondence is again encountered.

To begin, we establish a connection between conformal field theory and hyperbolic geometry in the language of the theory of phase transitions. However, it should be emphasized that our results are generally valid, a nd not dependent on this particular realization of the theory.

As demonstrated elsehwere [1], a domain boundary in the upper half-plane is created by boundary operators $\phi(x)[1-5]$ located at its endpoints on the real axis. These operators act to change the boundary condition along the edge of the system [3], the real axis. Boundary operators may also be defined by letting bulk operator in the system with a boundary approach the boundary, and making use of the bulk-boundary oeprtor product expansion $[1,4]$.

Although a domain boundary at a critical point exhibits large fluctuations, and has an energy that is not proportional to its length, it is a well-defined object. Conformal invariance implies universality, which allows one to study it in general. The (extra free) energy of such a boundary is

$$
F=-\ln \left\langle\phi\left(\mathrm{x}_{1}\right) \phi\left(\mathrm{x}_{2}\right)\right\rangle
$$

as described in [1]. For completeness, we note tht Equation (1) ignores both universal [3,6] and non-universal constants independent of $x_{1}, x_{2}$. The former are associated with the boundary states on the real axis, while the latter arise in computing the free energy of the boundary of any real system of statistical mechanical model.

Evaluating the correlation funciton, we find [1]

$$
F=2 \Delta \ln \left|\mathrm{x}_{1}-\mathrm{x}_{2}\right|,
$$

where $\Delta$ is the critical dimension of $\phi$. Now, Equation (1) also gives the domain boundary free energy in any geometry conformally equivlaent to the half-plane, if we evaluate the correlation function in the new geometry. This is done by making use of the basic equation of conformal covariance of correlation functions [7], as applied to boundary operators,

$$
\left\langle\phi_{1}\left(x_{1}\right) \phi_{2}\left(x_{2}\right) \ldots\right\rangle=\left|w\left(x_{1}\right)\right|^{\Delta_{1}}\left|w\left(x_{2}\right)\right|^{\Delta_{2}}\left\langle\phi_{1}\left(w_{1}\right) \phi_{2}\left(w_{2}\right) \ldots\right\rangle .
$$


Here $w=w(z)$ is an arbitrary conformal transformation, with $w_{i}=w\left(x_{1}\right)$.

Now, the domain boundary itself, in the half-plane, is a half circle betweent he points $x_{1}$ and $x_{2}$. This follows by considering a single change of boundary conditions, at the origin, say. By symmetry, the corresponding boundary lies along the $y$-axis. A projective transformation brings the endpoints to $x_{1}$ and $x_{2}$, and the straight line becomes a half circle. Such a half circle is precisely a geodesic of the hyperbolic, or Poincaré, metric $d s_{h}^{2}=\frac{1}{y^{2}} d s^{2}$ (for $y>0$ ) [8]. The tendency of the domain boundary to avoid the real axis corresponds to the divergence of the hyperbolic length as $y->0$.

Next consider the hyperbolic geodesic between the points $z_{1}=x_{1}+\varepsilon_{1}$, and $z_{2}=$ $x_{2}+\varepsilon_{2}$. Its hyperbolic length follows from standard results [8]

$$
l_{k}=2 \ln \left|\mathrm{x}_{1}-\mathrm{x}_{2}\right|-\ln \varepsilon_{1} \varepsilon_{2},
$$

and diverges as $z_{1}$ or $z_{2}$ approaches the real axis. On the other hand, it is natural to define the domain boundary to begin and end at a fintie but small (Euclidean) distance $\varepsilon$ above the real axis, where $\varepsilon$ will be on the order of the lattice spacing in any physical model. Then, up to additive constants, the boundary energy is proportional to the hyperbolic length,

$$
F=\Delta\left(l_{h}+\ln \varepsilon^{2}\right) .
$$

Note that factors giving rise to the $\ln \varepsilon^{2}$ term in Equation (5) will appear naturally in any lattice calculation of $F$ in a spin model, through the normalization of the conformal operators [9].

Next consider any other geometry that can be mapped to the half plane by a conformal transformation, for instance an infinite strip (with edges) of width $L, w=\frac{L}{\pi} \ln \mathrm{z}$. Under any such transformation, the hyperbolic length is invariant. The hyperbolic metric in the new geometry is induced by the transformation. In the strip, for instance, $g=\frac{(\pi / L)^{2}}{\sin ^{2}(\pi \nu / L)}$. Although the hyperbolic length of the boundary remains fixed, the transformation changes the distance between each endpoint and the edge of the system, by an amount proportional to the scale factor $|w(x)|$. For the transformed theory to represent a physical domain boundary in the new geometry, one must readjust its endpoints to be at distance $\varepsilon$ from the edges. Using the invariance mentioned and Equation (1) then leads directly to Equatiion (3), which is the basis of conformal field theory. The scale factors appear in (to our knowledge) an entirely new interpretation - they are necessary to insure that, in the new geometry, the boundary begins and ends in the appropriate place.

The argument of the preceding paragraph is not quite complete. The results described provide a hyperbolic interpretation for an arbitrary two-point correlation function of boundary operators. Specification of the full theory involves higher point correlation functions. The new element that appears is their dependence on cross-ratios [10]

$$
C=\frac{\left(x_{1}-x_{2}\right)\left(x_{3}-x_{4}\right)}{\left(x_{2}-x_{4}\right)\left(x_{1}-x_{3}\right)}
$$

However, if we consider the points $z_{i}=x_{i}+i \varepsilon$, it is easy to see that

$$
C=\exp \frac{1}{2}\left\{l_{h}(1,2)+l_{h}(3,4)-l_{h}(2,4)-l_{h}(1,3)\right\} .
$$


Now, as mentioned, $l_{h}$ is invariant under conformal transformations. The combination that appears in Equation (7) is, in addition, unaffected by the scale factors. Equation (3) thus follows immediately. It should be recognized that when more than one oeprator is included in the correlation function, a weighted sum of hyperbolic lengths will appear in the logarithm of the correlation function, with the weighting depending on the dimensions of the operators.

Note that in many cases four (and higher) point correlation functions can be interpreted in terms of domain boundary energies, including interactions [1]. In the case of critical percolation, an alternative interpretation of boundary operator correlation functions in terms of the probabilities of events is possible. This view has been exploited for the description of crossing probabilities in finite geometries [11-13].

The boundary operator theory is in general completely equivalent to the corresponding bulk conformal theory $[4,5,14]$, in the sense that, for a given central charge, the spectrum of primary operators is the same. However, a given operator will generally play a different role than in the bulk.

It should be emphasized that the systems to which the theory applies are defined in flat space. The hyperbolic geometry, which describes a space of constant negative curvature, arises naturally from the mathematics, without having been put in at the start, by contrast to other treatments of field theories [15] and statistical systems [16] defined on hyperbolic spaces. From a mathematical point of view, this is not completely unexpected. To represent a physical theory, the metric must be equivalent at equivalent points in the half-plane. Only two metrics satisfy this condition - Euclidean and hyperbolic. Of course this only means that the hyperbolic metric can occur, not that it necessarily will.

We pause to consider some implications of our results thus far. We have shown that the invariance of the hyperbolic length and natural physical requirements lead to a new derivation of the basic equation of conformal covariance, including a new interpretation of the scale factors. These considerations suggest that there is a fundamental mathematical connection between conformal field theory in the half-plane and hyperbolic geometry. Also, we have demonstrated that the energy of a domain boundary in any geometry conformally equivalent to the half-plane (e.g., Equation (5) of [1]) appears as a line integral along a hyperbolic geodesic. (In fact, it was precisely the search for a representation of the energy as a line integral that led to these results.) From a physical viewpoint, this is very surprising. At the critical point the boundary is strongly fluctuating - for instance, in a strip of width $L$, the boundary's width will be of the same order. There is absolutely no reason to expect that one of its intrinsic properties can be described as a line integral. A general picture of domain boundaries as independent, weakly interacting objects was established in [1]. The fact that the energy of a single boundary is proportional to its hyperbolic length, as described above, illuminates its nature further. More specifically, it indicates that a boundary, despite its large fluctuations, is in some sense additive.

Next consider an arbitrary three-point function. This has the form [7]

$$
\left\langle\phi_{l}\left(x_{1}\right) \phi_{m}\left(x_{2}\right) \phi_{n}\left(x_{3}\right)\right\rangle=\frac{C_{l m n}}{x_{21}^{\Delta_{l}+\Delta_{m}-\Delta_{n}} x_{32}^{\Delta_{m}+\Delta_{n}-\Delta_{l}} x_{31}^{\Delta_{l}+\Delta_{n}-\Delta_{m}}}
$$


where $C_{l m n}$ is an operator product expansion coefficient, $x_{j i}=x_{j}-x_{i}$, and we have taken $x_{1}<x_{2}<x_{3}$. Now consider the hyperbolic triangle defined by the three points $z_{i}=x_{i}+i \varepsilon$, $i=1,2,3$. Using the cosine law for hyperbolic triangles [8], it is then straightforward to show that the angles at points $x_{1}, x_{2}, x_{3}$ are of the form $a \varepsilon, b \varepsilon, c \varepsilon$, respectively, with $a=2$ $x_{32} / x_{21} x_{31}$, etc. It follows that

$$
\left\langle\phi_{l}\left(x_{1}\right) \phi_{m}\left(x_{2}\right) \phi_{n}\left(x_{3}\right)\right\rangle=C_{l m n}\left(\frac{a}{2}\right)^{\Delta_{l}}\left(\frac{b}{2}\right)^{\Delta_{m}}\left(\frac{c}{2}\right)^{\Delta_{n}} .
$$

Note the association of anomalous dimension and angle in Equation (9), and the fact that it is completely factored - each angle is raised to the power of the corresponding operator only, in contrast to Equation (8). Transforming Equation (9) to a new geometry, as above, reproduces the correct conformal covariance of the three-point function (Equation (3)) if one readjusts each vertex of the transformed triangle to be at distance $\varepsilon$ from the edge of the system.

If we let $\phi_{n}$ be the unit operator, Equation (9) describes the two-point function, by a triangle with a fictitious point $x_{3}$. The resulting expression is not independent of $x_{3}$ unless $\Delta_{l}=\Delta_{m}$, thus establishing orthogonality.

One can express the prefactor of an arbitrary four-point function $G$ as a product of four similar factors, by considering the hyperbolic quadrilateral defined by the four points taken at distance $\varepsilon$ above the real axis, as above. The remaining factor in $G$ is a function $\Phi$ of the cross-ratio $C$. This quantity may also be expressed through hyperbolic angles. If, for instance, one draws the triangle defined by points $x_{1}, x_{2}$, and $x_{3}, C$ is given by the ratio of the angle at $x_{3}$ of this triangle to the angle at $x_{3}$ of the quadrilateral.

Now consider a four-point function $G$ of operators $\phi$ degenerate at level two, i.e. $\phi_{(1,2)}$ or $\phi_{(2,1)}$. This condition implies that the dimension $\Delta$ of $\phi$ is an algebraic function of the central charge $c$, and that the factor $\Phi$ is proportional to a hypergeometric function, i.e. there is a factor in $G$ that satisfies a hypergeometric equation [10]. Now the ratio of two independent solutions of a hypergeometric equation defines the Schwarz function, which maps the upper half-plane onto a triangle with curvilinear sides [17, 18]. In the present case, the triangle is equiangular, with angle $\pi\left|\Delta^{\prime}\right|$, where $\Delta^{\prime}$ is the dimension of the operator $\phi^{\prime}$ (i.e. $\phi_{(1,3)}$ or $\phi_{(3,1)}$ ) appearing in the operator product expansion of $\phi$ with itself. Now one may reflect the triangle across any of its sides, which corresponds to a reflection of $C$ across the real axis. Repeating this procedure gives rise to a set of curvilinear triangles that may overlap, i.e. the inverse map is not necessarily single-valued. If we require single-valuedness, the triangles will tile (or, in the hyperbolic cases - see below, tessellate) a circular region [18]. This condition (for either choice of $\phi$ ) specifies $\Delta^{\prime}=|1|, 1= \pm 1, \pm 2, \pm 3, \ldots$. Since $\Delta^{\prime}$ determines $\Delta$, which in turn fixes the central charge, one arrives at the doubly-infinite discrete series

$$
c=1-3 \frac{(l-1)^{2}}{l(l+1)} .
$$

Equation (10) specifies a set of minimal models, including the Gassian model $(c=1, l=1)$, the Ising model $(c=1 / 2,1=2)$, critical percolation and dilute polymers $(c=0, l=3)$ 
[19], dense polymers [20] and matrix models [21-23] $(c=-2,|l|=\infty)$, and the Yang-Lee edge singularity $(c=-22 / 5, l=-5)[24]$. For $|l|>3$, the sum of the angles is less than $\pi$, so the triangles are hyperbolic, and a tessellation is produced. The inverse map is an automorphic function of the corresponding triangular group. For $|l|=1$, the triangle reduces to a great circle, and the group consists of one element. Similarly, $|l|=2$ gives the (finite) dihedral group $\langle 2,2,2\rangle$ [18]. Both of these cases correspond to spherical geometry, with total angle greater than $\pi$, while $|l|=3$ gives rise to a triangular lattice in flat space (total angle $\pi$ ). The inverse map is an automorphic function of the group so defined in each case.

In summary, we have established several connections between the conformal field theory of boundary operators and two-dimensional hyperbolic geometry. A new interpretation of the basic equation of conformal covariance arises, we find a fully factored form for the three-point function, and a doubly-infinite discrete series of central charges with limit $c=-2$ is discovered. A correspondence between the anomalous dimension and the angle of certain hyperbolic figures emerges.

P. K. is grateful for the hospitality of the School of Mathematics, Institute for Advanced Study, where part of this work was performed. I. V. acknowledges support from the Laboratory for Surface Science and Technology, University of Maine. We would like to thank I. Rivin for discussions and a particularly useful suggestion, and C. Callan, J. L. Cardy, K. Dawson, J. Distler, M. E. Fisher, C. Johnson, R. P. Langlands, E. Lieb, A. Ludwig, B. Nienhuis, A. Papadopoulos, J. H. H. Perk, I. Peschel, R. Rietman, P. Sarnak, T. Spencer, F. Williams and A. B. Zamolodchikov for stimulating conversations.

\section{References}

1. P. Kleban, Phys. Rev. Lett. 67, 2799 (1991).

2. H. W. Diehl and S. Dietrich, Z. Phys. B42, 65 (1981) [and Erratum 43, 281 (1981)].

3. J. L. Cardy, Nucl. Phys. B324, 581 (1989).

4. J. L. Cardy and D. C. Lewellen, Phys. Lett. B259, 274 (1991).

5. D. C. Lewellen, Nucl. Phys. B372, 654 (1992).

6. A. Ludwig and I. Affleck, Phys. Rev. Lett. 67, 161 (1991).

7. A. M. Polyakov, Pisma ZhETP 12, 538 (1970) [JETP Lett. 12, 381 (1970)].

8. A. F. Beardon, the Geometry of Discrete Groups, Graduate Texts in Mathematics 91 (Springer, Berlin, 1983).

9. J. L. Cardy, private communication.

10. A. A. Belavin, A. M. Polyakov and A. B. Zamolodchikov, Nucl. Phys. B241, 333 (1984). 
11. J. L. Cardy, J. Phys. A25, L201 (1992).

12. R. P. Langlands, C. Pichet, P. Oouliot, and Y. Saint-Aubin, J. Stat. Phys. 67, 553 (1992).

13. R. P. Langlands, P. Pouliot, and Y. Saint-Aubin, preprint.

14. D. C. Lewellen, private communication.

15. C. Callan and F. Wilczek, Nucl. Phys. B340, 366 (1990).

16. R. Rietman, B. Nienhuis and J. Oitmaa, preprint.

17. I. S. Gradshteyn and I. M. Ryzhik, Table of Integrals, Series, and Products, corrected ed. (Academic Press, Orlando, 1980).

18. M. Yoshida, Fuchsian Differential Equations, Aspects of Mathematics Vol. E11 (Vieweg, Braunschweig, 1987).

19. V. S. Dotsenko and V. A. Fateev, Nucl. Phys. B240, 312 (1984).

20. H. Saleur, Nucl. Phys. B382, 486 (1992).

21. E. Brézin and V. A. Kozakov, Phys. Lett. B236, 144 (1990).

22. M. Douglas and S. H. Shenker, Nucl. Phys. B335, 635 (1990).

23. D. J. Gross and A. A. Migdal, Nucl. Phys. B340, 333 (1990).

24. J. L. Cardy, Phys. Rev. Lett. 54, 1354 (1985). 